\title{
Augmented Two-Channel Arrhythmia Detection: An Efficient Diagnostic Method for Implantable Devices
}

\author{
CHIH-MING JAMES CHIANG, JANICE M. JENKINS, STEPHANIE A. CASWELL, \\ SHELLY A. STEVENSON, and LORENZO A. DICARLO \\ From the Department of Electrical Engineering and Computer Science School of Engineering, \\ University of Michigan, Ann Arbor; and The Michigan Heart and Vascular Institute and Cardiac \\ Electrophysiology Laboratory, St. Joseph Mercy Hospital, Ann Arbor, Michigan
}

CHIANG, C.-M.J., ET AL.: Augmented Two-Channel Arrhythmia Detection: An Efficient Diagnostic Method for Implantable Devices. ICDs are highly effective in preventing sudden cardiac death. However, inappropriate device shocks caused by false-positive diagnoses are estimated to happen in $20 \%$ of all patients. The need for implantable electrical devices to detect with precision arrhythmias requiring therapy has spawned a variety of proposals for better means of tachycardia identification. To address this problem, the augmented two-channel arrhythmia detection (A2CAD) algorithm, a real-time scheme utilizing timing and morphology from both the atrial and ventricular channels, is introduced. The algorithm uses rate detection as a first stage and augments this with morphological signal analysis in rhythms that confound the rate only diagnoses. The software executes in real-time (online), and has been tested on 60 passages of two-channel intracardiac signals. The following arrhythmias constituted the test set: $10 \mathrm{AF}$ and/or atrial flutter; 15 SVT; 16 VT; 10 ventricular flutter or VF; 5 sinus tachycardia; and 4 cases of AF concurrent with VF. Results from 60 patient cases indicate 57 (95\%) of 60 success rate for A2CAD, validating its potential for implementation in future implantable devices. (PACE 1996; 19:1493-1501)

arrhythmia, intracardiac electrogram, implantable devices, ICD, ventricular tachycardia, morphology

\section{Introduction}

Implantable cardioverter defibrillators (ICDs) have become popular choices of therapy for terminating potentially lethal tachyarrhythmias such as ventricular tachycardia (VT) and ventricular fibrillation (VF) with > 100,000 implantations since 1985. ${ }^{1}$ These devices have been shown to work well in the prevention of sudden cardiac death, with high sensitivity of detection of VTs and VFs. ${ }^{2}$ Third-generation devices with both pacing and

This work was partially supported by a grant from Medtronic Corporation, National Science Foundation Grants BCS8909042 and EID-9023514, and an equipment grant from Intel Corporation.

Address for reprints: Chih-ming J. Chiang, Ph.D., Telectronics Pacing Systems, 7400 S. Tucson Way, Englewood, CO 80112. Fax: (303) 799-2029.

Received September 12, 1994; revision July 10, 1995; accepted August 16, 1995. cardioversion-defibrillation capabilities have recently emerged with $>20,000$ implants. ${ }^{3}$ These devices, as those of earlier generations, rely upon ventricular sensing only, and continue to examine rate of ventricular depolarization as the major criterion of presence of VT or VF. These rudimentary detection schemes in earlier ICDs and third-generation ICDs with pacing options ${ }^{4,5}$ still lack specificity (false-positive detection of VT or VF), and inappropriate delivery of therapy remains a major problem. ${ }^{6-15}$

In this study, a heuristic and efficient algorithm is presented to solve the above mentioned problems. The schema, augmented two-channel arrhythmia detection (A2CAD), invokes statistically based decisions on groups of individual cardiac cycles and adds morphological analysis as necessary for diagnosis. This algorithm approximates methods used by human observers, who examine ECG signals in toto and derive a diagnosis in a global sense of underlying rhythm. 


\section{Methods}

\section{Bin Area Method}

Bin area method (BAM) ${ }^{16,17}$ is a time-domain template matching method for signal analysis. It compares bins (areas formed by groups of points) from a reference template with bins constructed from the signal to be analyzed. The value for each bin is calculated by the summation of consecutive samples of variable size $M$. Therefore, templates from $X$ number of points will have $X / M$ bins. Difference in the bins of the template and the signal to be analyzed is calculated, summed, and subtracted from one. The algorithm is not affected by baseline changes and amplitude fluctuations because it is normalized and average adjusted. Performance measures for BAM vary between -1 and 1 (with 1 being the best fit to the template).

The BAM was incorporated as the morphological method to reduce computation burden of morphological analysis. BAM was tested earlier offline on 25 passages from 18 patients, ${ }^{16,17}$ but has not heretofore been incorporated into an online analysis program. For this on-line study utilizing BAM morphological analysis, a 51-point template with 3-point bins was selected based upon empirical considerations and results.

\section{A2CAD}

The A2CAD system senses intracardiac atrial and ventricular electrograms, detects a fast rate in either chamber, and only then activates the main program for diagnosis of arrhythmia. A2CAD combines the rate scheme (which performs well in all non-1:1 arrhythmias) with BAM morphological analysis (which has the theoretical ability to separate many of the 1:1 cases such as sinus tachycardia [ST], supraventricular tachycardia [SVT], and VT with retrograde conduction). The algorithm detects arrhythmias within the constraints of realtime processing.

A2CAD was developed on an Intel X486/50E (Intel Corp, Hillsboro, OR, USA) with a Tecmar Labmaster (Scientific Solutions, Solon, OH, USA) analog-to-digital subsystem with 12-bit resolution. The programming language was $\mathrm{C}$. It was implemented for real-time execution and diagnosis on a cycle-by-cycle basis.

\section{Basic Structure of A2CAD}

A2CAD is composed of two main components, the data acquisition section and the analysis section, which is further divided into an initial interval detection and a more extensive morphological and interval diagnosis. The algorithm remains in a standby mode except for sensing the atrial and ventricular events at their appearance and then storing interval events into a buffer. Sensing is accomplished by a previously designed trigger that is adaptable to varying waveform amplitudes. ${ }^{18}$ Since morphological measures are sensitive to alignment of the waveforms being compared and therefore to the accuracy of the trigger, in the present study multiple BAM performance measures were calculated for each detected depolarization (varying the window around the trigger point) and the highest value chosen as the true measure.

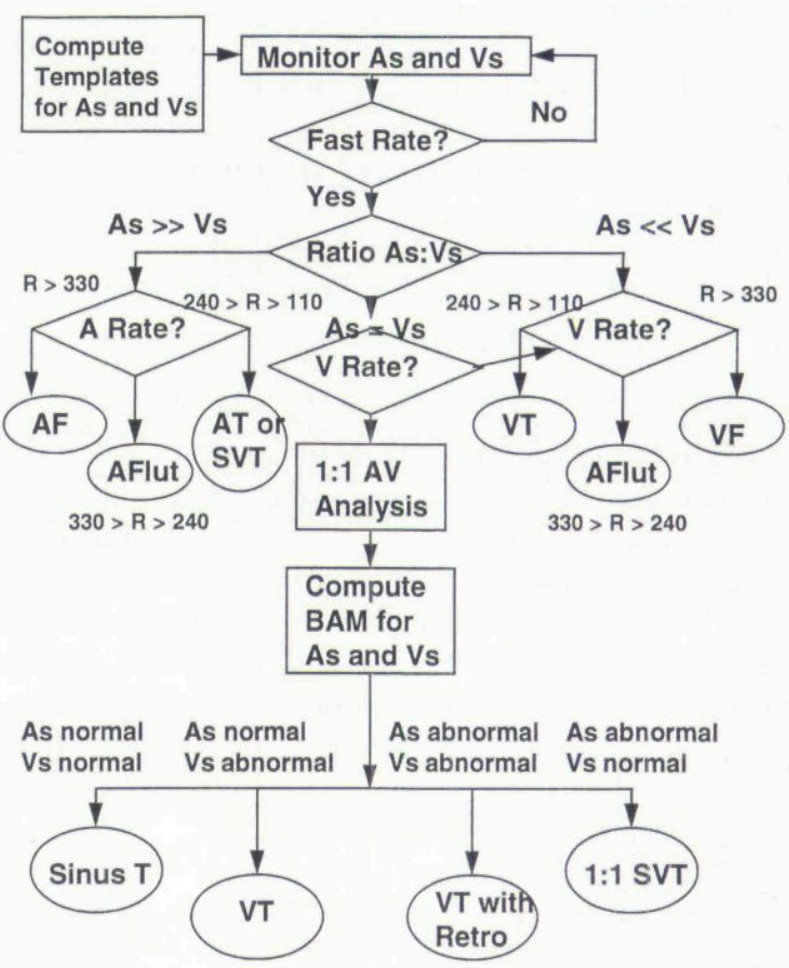

Figure 1. Diagnostic flowchart of $A 2 C A D . A=$ atrial; As $=$ atrial event $F=$ fibrillation $;$ Flut $=$ flutter $R=$ rate in cycles per minute; retro = retrograde; $S V T=$ supraventricular tachycardia; $T=$ tachycardia; $V=$ ventricular; $V s=$ ventricular event. 
Diagnostic Section of A2CAD

The analysis subsection of A2CAD is shown in Figure 1. Upon acquisition of the data, the average of the atrial-to-atrial (AA), atrial-to-ventricular $(\mathrm{AV})$, ventricular-to-atrial (VA), and ventricular-toventricular (VV) intervals for the last eight cycles (if $<8$, the total that is available) are computed and stored. If the intervals are normal, nothing further is done. If a sequence of short $(<545 \mathrm{~ms}, 6$ out of 8 consecutive cycles) intervals (fast rhythms) in either the atrial and/or ventricular chamber is detected, the program begins analysis. Initial logic determines if events in a tachycardia are predominantly atrial or ventricular based upon the ratio of atrial (A) to ventricular (V) events for the last 16 events. If the A to $\mathrm{V}$ ratio is $>1.5$, an atrial tachyarrhythmia is assumed and the atrial rate is examined. A diagnosis of atrial fibrillation (AF) is made if the atrial rate is $>330$ cycles per minute, and atrial flutter (AFlut) if the rate is between 240 and 330 cycles per minute. For a lower atrial rate, the diagnosis of atrial tachycardia (AT) with blocked conduction (or SVT) is made.

If the $\mathrm{V}$ to $\mathrm{A}$ event ratio is $>1.5$, the $\mathrm{VV}$ interval average is examined. If the rate exceeds $330 \mathrm{cy}-$ cles per minute, the diagnosis is VF. If between 240 and 330 cycles per minute, ventricular flutter (VFlut) is the diagnosis. For VFlut and VF, shock therapy will presumably be invoked. For slower rates, the VT diagnosis is given. Therapy for this rhythm varies depending on the underlying rate. Slow tachycardias are often well tolerated; faster ones are not.

The two side branches of the flow chart in Figure 1 is similar to that proposed by Arzbaecher et al. ${ }^{19}$ The two paths contain simple rate analysis for tachycardia diagnosis. The middle branch, where the number of $\mathrm{A}$ and $\mathrm{V}$ events are almost equal, represents the difficult diagnostic path: identification of 1:1 tachycardias. For this case, the algorithm first examines whether the ventricular rate is greater than the rate defined for flutter or fibrillation. If true, the rhythm is a competing $\mathrm{AF}$ and VF, or AFlut and VFlut, and the diagnostic logic defaults to VF or VFlut, respectively, since these are the more potentially lethal arrhythmias. If the ventricular rate is below flutter rate, both the $A$ and $V$ waveforms are compared to sinus rhythm (SR) templates using BAM for further analysis.
The next eight $\mathrm{A}$ and eight $\mathrm{V}$ waveforms are compared to stored A and V templates, created respectively from an earlier average of 20 sinus rhythm $\mathrm{A}$ and $\mathrm{V}$ waveforms of $51 \mathrm{~ms}$ (51 points with 17 3-point bins). Diagnosis of normal morphology is defined as 6 of 8 events in a particular chamber (to allow for ectopic cardiac cycles) classified above a fixed threshold of 0.70 of the BAM performance measure. For a normal morphology classification of both A and V events, ST is diagnosed. If the atrial waveform consistently falls into the normal category, and the $\mathrm{V}$ waveform is deemed abnormal, diagnosis is VT. Abnormal atrial and normal ventricular classification indicates 1:1 SVT. The remaining case, both abnormal atrial and abnormal ventricular classification, has the diagnosis of VT with retrograde conduction.

Results from processing each patient are shown in the following format: (1) depolarization number (all events, either A or V, are considered an event); (2) position in the signal file where triggers have occurred locating atrial depolarizations (these are sample point locations, but since we sample at $1,000 \mathrm{~Hz}$ they can be translated directly to milliseconds in the file); (3) position of triggers for ventricular depolarizations; (4) value of the BAM performance measure for A (0.00 indicates no morphology done); (5) value of BAM performance measure for $\mathrm{V}$ ( 0.00 indicates no morphology done); (6) average of AA interval (the last 8 unless there are fewer than 8 recorded); (7) duration of AV interval; (8) average of VV interval (the last 8 unless there are fewer than 8 recorded); (9) sequence type of the cycle, either $\mathrm{A}$ or $\mathrm{V}$ event; and (10) contextual rhythm diagnosis. When the diagnosis enters the 1:1 loop and has not completed morphology analysis, a temporary diagnosis of "Fast rhythm: checking" is reported.

A diagnosis for non-1:1 cases is derived from interval measurements of a stream of 16 cardiac events of either atrial or ventricular depolarizations or less if only fewer than 16 are available. Since the algorithm begins execution during the arrhythmia in all the test cases for this study, diagnosis is almost immediate for the non-1:1 cases. If the arrhythmia had begun spontaneously during processing, the diagnosis would require more intervals before confirming a fast rate. If the arrhythmia presented is $1: 1$ and is not a competing atrial/ventricular (concurrent) rhythm, then an 

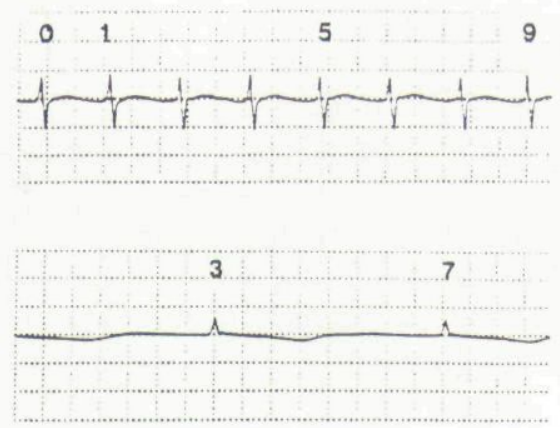

BEAT
0
1
2
3
4
5
6
7
8
9

\begin{tabular}{|c|c|c|c|c|c|c|c|c|}
\hline Apos & Vpos & BNMa & BNAtV & $\mathbf{M}$ & AV/VA & V $A$ & orv & Diagnosis \\
\hline 2168 & 2046 & 0.000 & 0.000 & 625 & .114 & 625 & a & S1nus Rhythm \\
\hline 2412 & 2046 & 0.000 & 0.000 & 625 & 114 & 625 & a & S1nus Rhyehm \\
\hline 2659 & 2046 & 0.000 & 0.000 & 247 & 114 & 625 & a & Sinus Rhythm \\
\hline 2659 & 2778 & 0.000 & 0.000 & 247 & 119 & 732 & $\nabla$ & SInus Rhythm \\
\hline 2912 & 2778 & 0.000 & 0.000 & 250 & 119 & 732 & a & Sinus Rhythm \\
\hline 3158 & 2778 & 0.000 & 0.000 & 249 & 119 & 732 & a & Atrial riutter \\
\hline 3401 & 2778 & 0.000 & 0.000 & 247 & 119 & 732 & a & Atrial Flutter \\
\hline 3401 & 3586 & 0.000 & 0.000 & 247 & 185 & 808 & $\nabla$ & Atrial Flutter \\
\hline 3652 & 3586 & 0.000 & 0.000 & 248 & 185 & 808 & a & Atrial rlutter \\
\hline 3897 & 3586 & 0.000 & 0.000 & 246 & 185 & 808 & a & Atrial rlutter \\
\hline
\end{tabular}

Figure 2. Example of a passage during atrial flutter. The top tracing for each of the next three figures is recorded from the high right atrial chamber of the heart (HRA), and the lower is a simultaneous recording taken from the right ventricular apex (RVA). Cardiac events ( $A$ and $V$ ) are numbered in chronological order. These event numbers have been superimposed onto the original recordings for visual interpretation. A portion of the diagnostic output is shown below the tracing. The horizontal axis of the tracing is 100 ser grid line, and the vertical axis $1 \mathrm{mV}$ per grid line. See text.

additional sequence of 16 events is needed for computing morphology. The beginning mean AA and VV intervals (events 0 and 1 ) have been artificially preset to $625 \mathrm{~ms}$ (a standard "normal" interval that is used for initialization). It typically requires two or three recognizable events in the same channel for the mean to be updated to actual intervals recognized by the computer. For examples of outputs, see Figures 2-4.

\section{Materials}

A2CAD was tested on bipolar intracardiac electrogram recordings obtained during electrophysiology studies. The signals were recorded on quadripolar electrode catheters (USCI Division C.R. Bard Inc., Billerica, MA, USA) with an interelectrode distance of $1 \mathrm{~cm}$. The catheters for the intraatrial and intraventricular electrogram recordings were positioned in the high right atrium or high right atrial appendage and in the right ventricular apex, respectively. The recordings were made on FM magnetic tape (Honeywell Electronics for Medicine, Denver, CO, USA) at a tape speed of 3.75 inches/s with 1- to $500-\mathrm{Hz}$ filtering. For A2CAD diagnosis, the FM tape was played, data digitized at $1,000 \mathrm{~Hz}$ per channel, and multichannel analysis was performed in realtime.

A total of 60 patient cases were tested with the following arrhythmia distribution: $10 \mathrm{AF}$ or flutter; 5 ST; 15 SVT; 10 VF or flutter; 16 VT; and $4 \mathrm{AF}$ concurrent with VF. The patient cases were chosen such that the algorithm would be thoroughly challenged. The length of the passages ranged from 10 seconds to 5 minutes.

Validation of the correct diagnosis was determined by overreading of all the passages with knowledge of patient history by an expert cardiologist blinded to the computer analysis.

\section{Results}

The results are shown in Table I. The initial rate only stage of the algorithm was sufficient for diagnosis in $36(60 \%)$ of 60 of the cases and mor-
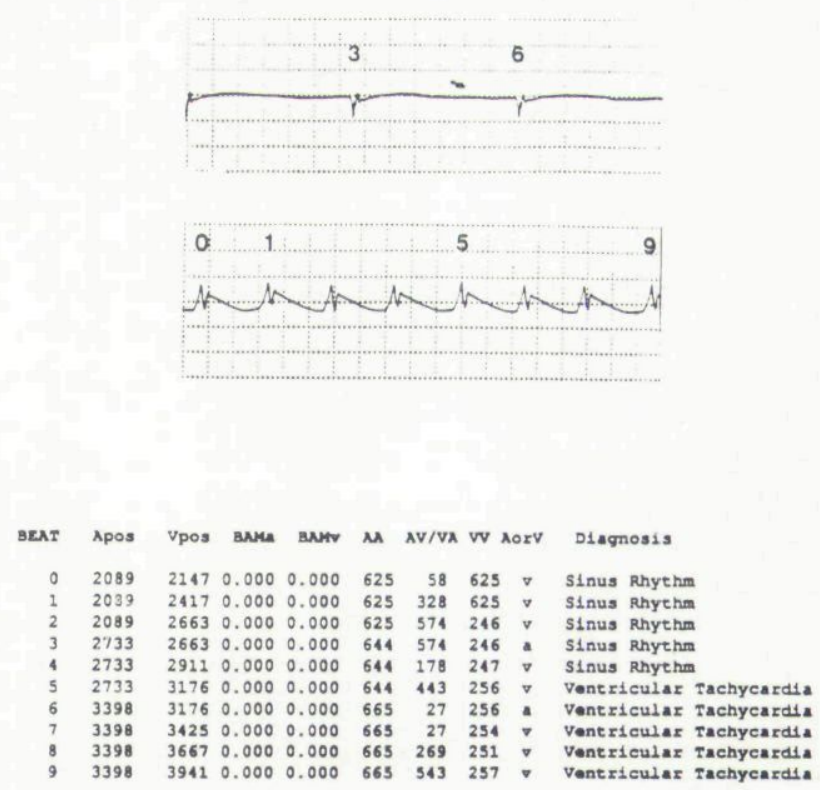

Figure 3. Example of a passage of ventricular tachycardia. For a full discussion of the figure please see text. 

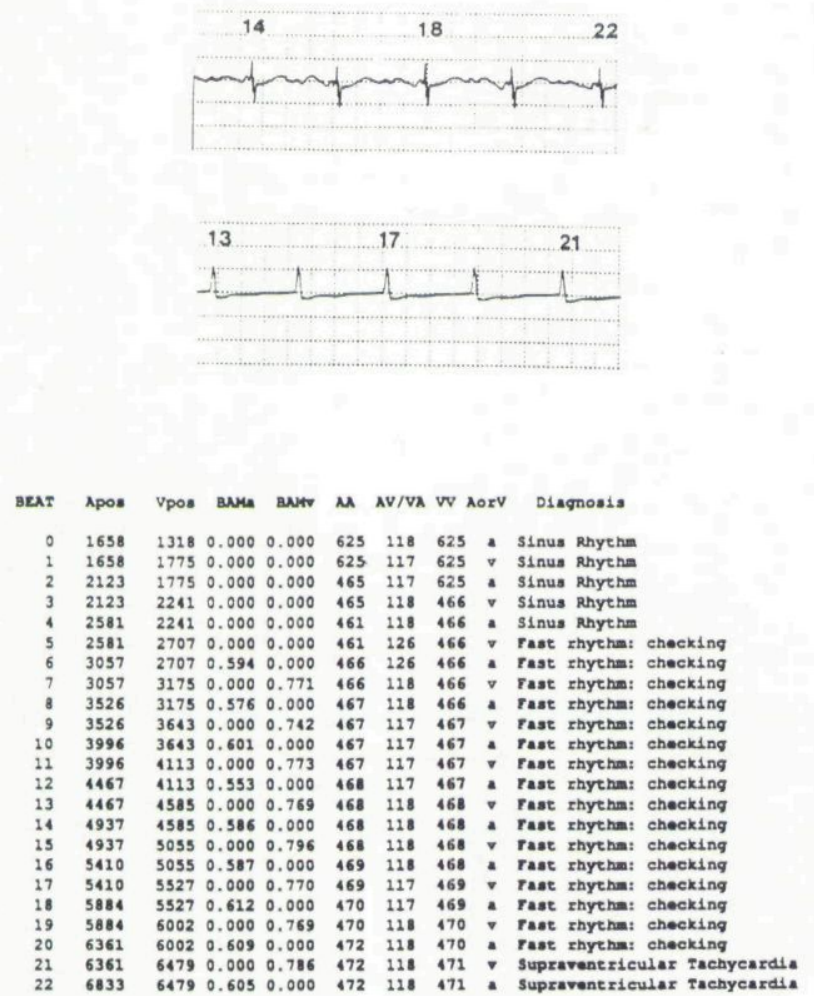

Figure 4. Example of a passage of 1:1 supraventricular tachycardia. This is a case of a 1:1 tachyarrhythmia that invoked morphology analysis to determine the underlying rhythm of SVT. For a full discussion of the figure please see text.

phological analysis was not invoked. In $12(20 \%)$ of 60 cases, the As were greater than Vs, with 10 yielding diagnosis of AF or AFlut, and 2 SVTs. In $20(33 \%)$ of 60 cases, Vs were greater than As, with resultant diagnoses of $10 \mathrm{VT}$ and 10 VFlut/VF. A2CAD obtained $32(100 \%)$ of 32 correct diagnoses for the above cases. The 4 remaining cases of the 36 episodes exhibited competing rhythms of either AF and VF, or AFlut and VFlut. All four were all diagnosed correctly in terms of the ventricular rhythm. Overall, the rate analysis A2CAD achieved $36(100 \%)$ of 36 accuracy.

The distribution of the remaining 24 cases with 1:1 atrioventricular (AV) relationship above the tachycardia rate (which automatically invoked BAM) is as follows: 5 ST (4/5 diagnosed correctly with normal $\mathrm{A}$ and $\mathrm{V}$ waveforms and 1/5 diagnosed incorrectly as SVT because of the misclassification of the A waveform); 13 SVT (11/13 diagnosed correctly with abnormal A and normal V waveforms and 2/13 diagnosed incorrectly as VT with retrograde because of misclassification of the V waveform); and 6 VT with retrograde conduction (6/6 diagnosed correctly with abnormal A and V waveforms). A2CAD gave 21 ( $88 \%$ ) of 24 correct diagnoses for the 1:1 AV cases in the tachycardia range. Overall, A2CAD had a $95 \%$ success rate or 57 of 60 patient cases correct.

The dramatic improvement of A2CAD over strictly rate methods without sudden onset or sustained rate criteria is evident. Although A2CAD did not achieve $100 \%$ success in the 1:1 cases, it performs better than rate only sensing schemes that would misdiagnose all the ST and SVT cases as VT with $6(25 \%)$ of 24 accuracy.

Exemplary passages of A2CAD processing are shown in Figures 2-4 with a portion of the processed signals. One sample from each of the arrhythmias is presented with an abbreviated portion of the computer report. For explanation of the output, see the Methods section.

Figure 2 is an example of a passage of AF. Three short A events (Nos. 2, 4, and 5) with concomitant normal ventricular events (\#3 and later \#7) give rise to an atrial flutter diagnosis upon event 5 . The atrial intervals fall within the range of 247-250 ms, which meets the requirement of a flutter heart rate of 240-330 cycles per minute, i.e., intervals in the range of $182-250 \mathrm{~ms}$. No morphology measure is invoked.

Figure 3 is an example of a passage of VT. A sequence of $\mathrm{V}$ events numbers 2,4 , and 5 , which range from 246-256 ms (and occur with a relationship of 2:1 or 3:1 with respect to A events), meets the test for a VT. Ventricular intervals fall within the $<240$ cycles per minute rate (i.e., intervals $>250 \mathrm{~ms}$ ). The atrial rate is entirely within the normal range ( $>545 \mathrm{~ms}$ ). (See A events numbers 3 and 6 ).

Figure 4 is an example of a passage of 1:1 SVT. This is a case of 1:1 tachyarrhythmia that invoked morphology analysis to determine the etiology. At event number 5, the computer recognizes a fast rhythm with a 1:1 A to $\mathrm{V}$ relationship and a non-VF or flutter rate, and proceeds to initiate waveform analysis using BAM. Sixteen events after the start, due to abnormal waveform diagnosis in the atrial channel $\left(\mathrm{BAM}_{\mathrm{a}}\right.$ values below the 0.70 threshold) and normal waveform diagnosis of ventricular waveforms $\left(\mathrm{BAM}_{\mathrm{v}}\right.$ values $\left.>0.70\right)$, the di- 
Table I.

A2CAD (BAM) Results of 60 Test Cases of Arrhythmia Diagnosis

\begin{tabular}{|c|c|c|c|c|c|c|c|c|c|}
\hline Case & Diagnosis & Correct & $\begin{array}{l}\text { A Cycle } \\
\text { Length }\end{array}$ & $\begin{array}{l}\text { V Cycle } \\
\text { Length }\end{array}$ & Case & Diagnosis & Correct & $\begin{array}{l}\text { A Cycle } \\
\text { Length }\end{array}$ & $\begin{array}{l}\text { V Cycle } \\
\text { Length }\end{array}$ \\
\hline 1 & VT $1: 1$ ret & Y & 402 & 402 & 32 & 1:1 SVT & Y & 360 & 360 \\
\hline 2 & VT $1: 1$ ret & Y & 302 & 302 & 33 & VT $1: 1$ ret & Y & 340 & 340 \\
\hline 3 & VT $1: 1$ ret & Y & 402 & 402 & 34 & AFlut & Y & $186-236$ & $650-799$ \\
\hline 4 & AFlut & $Y$ & 202 & 404 & 35 & VT $1: 1$ ret & $Y$ & 330 & 330 \\
\hline 5 & $\mathrm{AF}$ & Y & $125-160$ & $690-835$ & 36 & $1: 1$ SVT & Y & 463 & 463 \\
\hline 6 & AFlut & Y & 248 & $732-938$ & 37 & $1: 1 \mathrm{SVT}$ & $\mathrm{N}$ & 286 & 286 \\
\hline 7 & $3: 1 \mathrm{SVT}$ & Y & 300 & $825-999$ & 38 & VT & $Y$ & 670 & $317-366$ \\
\hline 8 & $1: 1$ SVT & Y & 370 & 370 & 39 & VT & Y & 718 & 320 \\
\hline 9 & ST & Y & 501 & 501 & 40 & VFlut & Y & $658-749$ & 240 \\
\hline 10 & $\mathrm{AF}$ & Y & 180 & $613-782$ & 41 & VT & Y & 650 & 260 \\
\hline 11 & AFlut & Y & $182-203$ & $451-758$ & 42 & VT & $\mathrm{Y}$ & 812 & $270-314$ \\
\hline 12 & SVT & Y & $465-485$ & $465-485$ & 43 & VT & Y & $751-808$ & $420-515$ \\
\hline 13 & AFlut & Y & $200-227$ & $450-804$ & 44 & VFlut/F & Y & $415-784$ & $177-221$ \\
\hline 14 & $1: 1 \mathrm{SVT}$ & Y & 272 & 272 & 45 & VT & Y & $823-867$ & 395 \\
\hline 15 & $1: 1 \mathrm{SVT}$ & Y & 480 & 480 & 46 & $\mathrm{AF} / \mathrm{VF}$ & Y & $169-197$ & $165-201$ \\
\hline 16 & $1: 1 \mathrm{SVT}$ & $\mathrm{N}$ & 446 & 446 & 47 & $\mathrm{AF} / \mathrm{VF}$ & Y & $180-232$ & $176-189$ \\
\hline 17 & VT & $Y$ & $1134-1198$ & 444 & 48 & VT & $Y$ & 790 & $343-390$ \\
\hline 18 & VT & Y & $870-1010$ & 425 & 49 & VFlut & Y & $577-618$ & $183-191$ \\
\hline 19 & VFlut & $Y$ & $677-702$ & $197-347$ & 50 & AFlut & $\mathrm{Y}$ & $198-232$ & $625-1176$ \\
\hline 20 & VFlut (Fib) & $\mathrm{Y}$ & $672-767$ & $151-201$ & 51 & $\mathrm{AF}$ & Y & $168-182$ & $368-419$ \\
\hline 21 & VF & $\mathrm{Y}$ & $363-455$ & $157-210$ & 52 & VFlut & Y & $416-695$ & $204-230$ \\
\hline 22 & $2: 1 \mathrm{SVT}$ & $Y$ & $272-310$ & $551-686$ & 53 & VFlut/F & $Y$ & $652-671$ & $173-190$ \\
\hline 23 & $1: 1 \mathrm{SVT}$ & $\mathrm{Y}$ & 303 & 303 & 54 & VFlut & Y & $668-692$ & $194-205$ \\
\hline 24 & $1: 1 \mathrm{SVT}$ & Y & 520 & 520 & 55 & $\mathrm{AF} / \mathrm{VF}$ & $Y$ & $103-122$ & $122-133$ \\
\hline 25 & VFlut & Y & $702-920$ & $207-220$ & 56 & AFlut/VFlut & Y & $254-281$ & $198-232$ \\
\hline 26 & VT $1: 1$ ret & Y & 500 & 500 & 57 & $1: 1 \mathrm{ST}$ & $\mathrm{N}$ & $450-455$ & $450-455$ \\
\hline 27 & 1:1 SVT & $Y$ & 395 & 395 & 58 & $1: 1 \mathrm{ST}$ & $Y$ & $451-525$ & $451-525$ \\
\hline 28 & $1: 1 \mathrm{SVT}$ & Y & 510 & 510 & 59 & $1: 1 \mathrm{ST}$ & Y & $478-491$ & $478-491$ \\
\hline 29 & $1: 1$ SVT & Y & 305 & 305 & 60 & $1: 1 \mathrm{ST}$ & Y & $448-534$ & $448-534$ \\
\hline 30 & AFlut/F & $Y$ & $167-219$ & $719-1073$ & 60 total & & 57 correct & & \\
\hline 31 & VT & Y & $777-798$ & $433-452$ & & & & & \\
\hline
\end{tabular}

Correct 57 out of $60(95 \%)$.

$\mathrm{A}=$ atrial; $\mathrm{F}=$ fibrillation; Flut $=$ flutter; $\mathrm{n}: \mathrm{n}=\mathrm{A}: \mathrm{V}$ relationship; ret $=$ retrograde; $\mathrm{SV}=$ supraventricular; $\mathrm{T}=$ tachycardia; $\mathrm{V}=$ ventricular.

agnosis is SVT, another arrhythmia that the device must recognize. See lower rightmost terminal point of the flow chart in Figure 1 for the diagnosis. In the interest of space, tracings again show only the latter part of the diagnostic output from events nos. 11-22.

\section{Discussion and Future Work}

Morphological signal analysis methods have been proposed to address the specificity problem, among them frequency- and time-domain techniques. ${ }^{16,17,20-31}$ Since frequency-domain methods have been inconclusive in discriminating VT from SR, time-domain template matching methods have developed, among them the classic correlation waveform analysis (CWA), ${ }^{29-31}$ and other fast algorithms such as BAM. ${ }^{16,17}$ All have been tested on intracardiac electrograms and shown to perform well in improving specificity of diagnosis. 
Aside from morphological analysis, utilizing information from the atrial channel (dual chamber rate analysis) has also been proposed for alleviating the false-positive shock problem. Arzbaecher et al. ${ }^{19}$ demonstrated that two chamber rate analysis was superior to single chamber analysis for intracardiac leads and tested it on 22 patients. In all cases where atrial depolarizations exceeded ventricular depolarizations in the presence of a fast rate, or where ventricular depolarizations exceeded atrial, the diagnosis was straightforward and accurate. For the case of 1:1 arrhythmias, the algorithm was invoked in nine cases and failed in one. The use of an onset criterion separated ST (normal accelerated heart rate) from pathological tachycardia, but was unable to distinguish SVT from VT with retrograde activation. Thus, the 1:1 rhythms still remain the most difficult to separate even in the case of two-channel signal acquisition. Concurrent atrial and ventricular tachyarrhythmias also present problems.

A detection scheme utilizing the combination of dual chamber rate analysis with the addition of dual chamber morphological signal analysis was first proposed by DiCarlo et al. ${ }^{32}$ The algorithm executed as an off-line program and was tested on an atrial esophageal lead and surface lead and intended for application to coronary care monitoring. Its internal logic was redesigned for modification as a real-time arrhythmia detection scheme and was renamed the 2-Channel Rate Morphology (2CRM) Method. ${ }^{33}$ It was tested on-line on twochannel intracardiac signals. However, 2CRM attempts to associate each $\mathrm{A}$ event with a $\mathrm{V}$ event and fails in distinguishing dissociated $\mathrm{A}$ and $\mathrm{V}$ events that are common in VT and VF. Another proposed method for combining dual chamber analysis and morphological analysis using neural networks ${ }^{34}$ has been proposed. It has several key drawbacks, such as requiring human feedback to determine the existence of 1:1 arrhythmias, inability to distinguish between ST and SVT, and execution is limited to an off-line mode at present.

In this study, A2CAD, a new and more efficient real-time arrhythmia detection scheme is presented. It borrows from the earlier two-channel method (more atrial than ventricular events, or more Vs than As) for determination of a majority of arrhythmias, ${ }^{19}$ but contains a supplementary morphological metric for separating the more complex arrhythmias that do not fall into the simple categories. The morphometric used is a fast algorithm (BAM) that replicates the correlation method at a fraction of the computational cost.

Results from 60 patients gave a performance of over 95\% success, with much better 1:1 tachyarrhythmia discrimination than current detection schemes for implantable devices. By adding a morphological measure, A2CAD has dramatically improved the specificity of detection from current ICD devices with or without pacing options. Rate only detection schemes without sudden onset or sustained rate would have classified all the 1:1 tachycardia cases as VT (or 6 [25\%] of 24 correct), while A2CAD obtained $21(88 \%)$ of 24 correct. Therefore, the value of this study has been demonstrated in a great reduction in false-positive detection of VT while maintaining sensitivity performance (VT/VF detection) as shown by the patient results.

One of the potential limitations of the present study was the examination of bipolar electrograms only. Whether the results might differ for unipolar or more widely spaced catheter recordings is unknown. The effect of drugs or degenerative conditions could also change the morphology of waveforms, and temporal updating of the templates created during SR would be required in actual implementation. Since the recordings were obtained from acute rather than chronic leads, there may be more variation in the signals examined during this study than from a patient with catheters permanently affixed to the myocardium. Conversely, the signals were obtained during electrophysiology studies while patients were supine and sedated and might contain less variability than that present in active, ambulatory subjects.

The potential ambiguities persisting in morphological analysis remain as shown in the following cases: (1) ambiguity in the diagnosis of ST versus paroxysmal SVT or VT. A preliminary study using the median as a sudden onset criterion to address this problem has been published ${ }^{35}$; and (2) ambiguity in the diagnosis of SVT with aberrant conduction or VT with retrograde ${ }^{36}$ as evidenced by the two erroneous diagnosis made by A2CAD. The probability of rate related aberrancy with paroxysmal bundle branch block belongs in this category. Only $5.3 \%$ of all VT cases would fall under this classification. ${ }^{36}$ The algorithm would 
misclassify these cases as VT, but the probability of 1:1 VT conduction ${ }^{37,38}$ from $15 \%-22 \%$ combined with the probability of rate related aberrancy would mean a false-positive detection of $1.1 \%$. However, a proposed solution examining the sequence of $\mathrm{A}$ or $\mathrm{V}$ events is presented in a separate study by Chiang et al. ${ }^{39}$

Therefore, by utilizing morphological analysis, A2CAD dramatically improved specificity of performance, but $100 \%$ performance is still not achievable. Perhaps a combination of the newly proposed improvements along with morphological analysis in A2CAD would further improve the performance of the algorithm.

Another specificity problem for implantable devices that remains unsolved by A2CAD is discrimination between $\mathrm{AF}$ with fast ventricular response and VT with competing AF or AFlut. Current devices would classify both as VT, but A2CAD defaults to the faster rate and would therefore diagnose both cases as AF or AFlut. A potential solution is proposed in which rate regularity

\section{References}

1. Cardiac imaging, dual-chamber defibrillators fuel market growth. Cardiovascular Device Update 1996;2:1-3.

2. Cohen T, Chien W, Lurie K, et al. Implantable cardioverter defibrillator proarrhythmia: Case report and review of the literature. PACE 1991; 14:1326-1329.

3. Olson W. Medtronic, Inc., August 1993, personal communication.

4. Haluska E, Whistler S, Calfee R. A hierarchical approach to the treatment of ventricular tachycardias. PACE 1986; 9:1320-1324.

5. Pless BD, Sweeney M. Discrimination of supraventricular tachycardia from sinus tachycardia of overlapping cycle length. PACE 1984; 7:1318-1324.

6. Platia E, Griffith L, Reid P. Complications with the automatic implantable cardioverter defibrillator. (abstract) J Am Coll Cardiol 1986; 2:200.

7. Chapman P, Troup P. The automatic implantable cardioverter-defibrillator: Evaluating suspected inappropriate shocks. J Am Coll Cardiol 1987; 7:1075-1078.

8. Kim S, Furman S, Matos J, et al. Automatic implantable cardioverter defibrillator: Inadvertent discharges during permanent pacemaker magnet tests. PACE 1987; 10:579-582.

9. Manz M, Gerckens U, Lüderitz B. Erroneous dis- for VT and multiplicity relationships between the atrial and ventricular intervals are utilized to separate VT from fast ventricular response in $\mathrm{AF}$ and AFlut. ${ }^{40}$

Further refinements in automated analysis warrant additional examination. One potential refinement is the effect of filtering of the electrograms on the performance of A2CAD. All intracardiac electrograms were tested from wideband signal settings $(1-500 \mathrm{~Hz})$ and at a $1,000-\mathrm{Hz}$ sampling rate. A more narrow passband with lower sampling rate would increase computational efficiency. A previous study has shown that moderate filtering does not affect the results of our morphological metric (BAM). ${ }^{41}$ Addition of this feature could further optimize future versions of A2CAD.

After examining the performance due to these factors, the proposed improvements discussed in this section (median filter, onset sequence, rate regularity, and multiplicity) could be incorporated into $\mathrm{A} 2 \mathrm{CAD}$ to further improve diagnostic accuracy.

charge from an implanted automatic defibrillator during supraventricular tachyarrhythmia induced ventricular fibrillation. Am J Cardiol 1986; 57:343-344.

10. Steinberg J, Sugalski J. Cardiac rhythm precipitating automatic implantable cardioverter-defibrillator discharges in outpatients as detected from transtelephonic electrocardiographic recordings. Am J Cardiol 1996; 67:95-97.

11. Grimm W, Flores B, Marchlinski F. Electrocardiographically documented unnecessary, spontaneous shocks in 241 patients with implantable cardioverter-defibrillators. PACE 1992; 15:16671673.

12. Newman D, Dorian P, Downar E, et al. Use of telemetry functions in the assessment of implanted antitachycardia device efficacy. Am J Cardiol 1992; 70:616-621.

13. Schmitt C, Montero M, Melichercik J. Significance of supraventricular tachyarrhythmias in patients with implanted pacing cardioverter defibrillators. PACE 1994; 17:295-302.

14. Sandler M, Kutalek. Inappropriate discharge by an implantable cardioverter defibrillator: Recognition of myopotential sensing using telemetered intracardiac electrograms. PACE 1994; 17:665-671.

15. Hamer M, Clair W, Wilkinson W, et al. Evaluation 
of outpatients experiencing implantable cardioverter defibrillator shocks associated with minimal symptoms. PACE 1994; 17:938-943.

16. Throne R, Jenkins J, DiCarlo L. The bin area method: A computationally efficient algorithm for analysis for ventricular and atrial intracardiac electrograms. PACE 1990; 13:1286-1297.

17. Throne R, Jenkins J, DiCarlo L. A comparison of four new time-domain techniques for discriminating monomorphic ventricular tachycardia from sinus rhythm using ventricular waveform morphology. IEEE Trans Biomed Eng 1991; 38:561-570.

18. McDonald R, Jenkins J, Arzbaecher R, et al. A software trigger for intracardiac waveform detection with automatic threshold adjustment. IEEE Comp Cardiol. New York, NY, IEEE Press, 89CH2932-2.

19. Arzbaecher R, Bump T, Jenkins J, et al. Automatic tachycardia recognition. PACE 1984; 7(II):541547.

20. Davies D, Wainwright R, Tooley M, et al. Detection of pathological tachycardia by analysis of electrogram morphology. PACE 1986; 9:200-208.

21. Langberg J, Gibbs W, Auslander D, et al. Identification of ventricular tachycardia with use of the morphology of the endocardial electrogram. Circulation 1988; 77:1363-1369.

22. Pannizzo F, Furman S. Frequency spectra of ventricular tachycardia and sinus rhythm in human intracardiac electrograms: Application to tachycardia detection for cardiac pacemakers. IEEE Trans Biomed Eng 1988; 37:421-425.

23. Tomaselli G, Nielsen A, Finke W, et al. Morphologic differences of the endocardial electrogram in beats of sinus and ventricular origin. PACE 1988; 11:254-262.

24. Slocum J, Sahakian A, Swiryn S. Computer discrimination of atrial fibrillation and regular atrial rhythms from intra-atrial electrograms. PACE 1988; 11:610-621.

25. Ripley K, Bump T, Arzbaecher R. Evaluation of techniques for recognition of ventricular arrhythmias by implanted devices. IEEE Trans Biomed Eng 1989; 36:618-624.

26. Ropella K, Baerman J, Sahakian A, et al. Differentiation of ventricular tachyarrhythmias. Circulation 1990; 82:2035-2043.

27. Steinhaus B, Wells R, Greenhut S, et al. Detection of ventricular tachycardia using scanning correlation analysis. PACE 1990; 13:1930-1936.

28. Paul V, O'Nunain S, Malik M, et al. Temporal electrogram analysis: Algorithm development. PACE 1990; 13:1943-1947.

29. Lin D, DiCarlo L, Jenkins J. Identification of ven- tricular tachycardia using intracavitary ventricular electrograms: Analysis of time and frequency domain patterns. PACE 1988; 11:1592-1605.

30. DiCarlo L, Throne R, Jenkins J. A time-domain analysis of intracardiac electrograms for arrhythmia detection. PACE 1991; 14:329-336.

31. Throne R, Jenkins J, Winston S, et al. Discrimination of retrograde from anterograde atrial activation using intracardiac electrogram waveform analysis. PACE 1989; 12:1622-1630.

32. DiCarlo L, Lin D, Jenkins J. Automated interpretation of cardiac arrhythmias: Design and evaluation of a computerized model. J Electrocardiol 1993; 26:53-67.

33. Chiang CJ, Jenkins J, DiCarlo L. Real-time arrhythmia identification from automated analysis of intraatrial and intraventricular electrograms. PACE 1993; 16:223-227.

34. Leong P, Jabri M. MATIC-An intracardiac tachycardia classification system. PACE 1992; 15:1317-1331.

35. Chiang CJ, Jenkins J, DiCarlo L. Discrimination of ventricular tachycardia from sinus tachycardia by antitachycardia devices: Value of median filtering. Med Eng Phys 1994; 16:513-517.

36. Throne R, DiCarlo L, Jenkins J, et al. Paroxysmal bundle branch block of supraventricular origin: A possible source of misdiagnosis in detecting ventricular tachycardia using time domain analyses of intraventricular electrograms. PACE 1990; 13:453-468.

37. Li HG, Zardini M, Thakur RK, et al. Ventriculoatrial conduction in patients with implantable cardioverter-defibrillators: Implications for tachycardia discrimination by dual chamber sensing. Circulation 1993; 88:I54.

38. Schuger CD, Jackson K, Steinman RT, et al. Atrial sensing to augment ventricular tachycardia detection by the automatic implantable cardioverter defibrillator: A utility study. PACE 1988; 11:1456-1464.

39. Chiang CJ, Jenkins J, DiCarlo L. Value of onset sequence in discriminating ventricular tachycardia from supraventricular tachycardia. IEEE Comp Cardiol. New York, NY, IEEE Press, 93CH3384-5.

40. Chiang CJ, Jenkins J, DiCarlo L. The value of rate regularity and multiplicity measures to detect ventricular tachycardia in the presence of atrial fibrillation or flutter. PACE 1994; 17:1503-1508.

41. Chiang C, Jenkins J, DiCarlo L. Impact of filtering upon ventricular tachycardia identification by correlation waveform analysis. PACE 1991; 14:1809-1814. 
This document is a scanned copy of a printed document. No warranty is given about the accuracy of the copy. Users should refer to the original published version of the material. 